Published in "Polymer Chemistry 10(16): 2057-2063, 2019"

which should be cited to refer to this work.

\title{
Synthesis and properties of poly(norbornene)s with lateral aramid groups $\uparrow$
}

\author{
Phally Kong, $\sharp^{\mathrm{a}}$ Susanne Drechsler, $t^{\mathrm{a}}$ Sandor Balog, (D) ${ }^{\mathrm{b}}$ Stephen Schrettl, (D) ${ }^{\mathrm{b}}$ \\ Christoph Weder (D) ${ }^{b}$ and Andreas F. M. Kilbinger (DD *a
}

\begin{abstract}
This paper deals with the synthesis and investigation of comb-like poly(norbornene)s carrying lateral rod-like aramid groups. Two types of norbornene-based monomers were synthesized and copolymerized with a norbornene carrying an aliphatic side chain using ring opening metathesis polymerization (ROMP). The new monomers contain aramid derivatives that display different types of non-covalent interactions. The first monomer contains linear tri( $p$-benzamide)s, which exhibit the typical $\mathrm{H}$-bonds that aramids are known for. The second monomer features tri( $p$-benzamide)s with bulky ethylhexyloxy side-chains, which suppress intermolecular hydrogen bonding and favor $\pi-\pi$-stacking. The monomers were copolymerized in various ratios and the influence of the composition on the material properties was investigated using Differential Scanning Calorimetry (DSC), Thermogravimetric Analysis (TGA), Dynamic Mechanical Analysis (DMA), and powder X-ray diffraction (XRD) experiments. The results show that the glass transition temperature increases proportionally with the concentration of the $\mathrm{H}$-bonding monomer
\end{abstract}

\section{Introduction}

Aramids are known to be very resistant materials and are known under tradenames such as Kevlar ${ }^{\circledR}$ and Nomex®. The high stiffness of these materials is a result of non-covalent interactions, such as H-bonds. ${ }^{1-3}$ Responsible for the attractive material properties on the one side, these interactions also cause drawbacks such as poor solubility and hence processability. ${ }^{4}$ One possibility to overcome this disadvantage is the attachment to soluble polymer chains. ${ }^{5,6}$ To date, research within the field of comb polymers focused mostly on rod-like polymers with hairy side chains ${ }^{7,8}$ or on rigid rod-like side chains connected via a flexible linker to the main chain. ${ }^{9}$ Only few reports exist on theoretical studies ${ }^{10}$ and syntheses ${ }^{11}$ of polymers in which the rod-like side chain is directly bound to the main chain. Furthermore, these reports mainly focused on using terephthalic acid, polyphenyl, or phenyl alkynyl oligomers as rigid rods. ${ }^{12,13}$ The aim of this work was the synthesis

${ }^{a}$ University of Fribourg, Chemistry Department, Rue du Musée 9, 1700 Fribourg, Switzerland.E-mail: andreas.kilbinger@unifr.ch

${ }^{b}$ Adolphe Merkle Institute, University of Fribourg, Chemin des Verdiers 4, 1700 Fribourg, Switzerland

$\dagger$ Electronic supplementary information (ESI) available: Syntheses, NMR spectra verifying DMB-deprotection, DLS data, GPC data, NMR calculating actual molar ratio of rod content, complete DSC curves, complete tensile stress-strain-test data and POM photographs. See DOI: 10.1039/c9py00187e

$\ddagger$ These authors contributed equally. of comb-like poly(norbornene)s carrying lateral rod-like aramid groups and the investigation of how the properties of such materials can be controlled by exploiting different types of non-covalent interactions, which are a powerful tool for supramolecular organization. In this paper, we present the synthesis of statistical copolymers made from norbornenebased monomers carrying either tri ( $p$-benzamide) rods or aliphatic side chains. These comb-like polymers were prepared via ring-opening metathesis polymerization (ROMP). Aramids are good candidates to undergo different supramolecular interactions, such as hydrogen bonds ${ }^{14}$ and aromatic stacking $^{15,16}$ In order to investigate the distinct influence of either one of these intermolecular forces, we synthesized two types of monomers containing different rigid rod-like components. One monomer possesses a rod consisting of an aramid trimer able to interact primarily via hydrogen bonds. In contrast, the second monomer contains a rigid element, through which $\pi$-interactions are more likely to occur. The monomers were copolymerized in various ratios and the influence of the composition on the mechanical and thermal properties was investigated. It was found that hydrogen bonding between the side chains increased the glass transition temperature significantly. Furthermore, it was observed that these interactions were also beneficial for the mechanical properties, resulting in polymers with a higher storage modulus. The findings of this study might serve as blueprints to improve the properties of other polymeric systems. 


\section{Results and discussion}

The monomer synthesis started with the thermal conversion of commercially available cis-5-norbornene-endo-2,3-dicarboxylic anhydride to the exo-form. Subsequent reaction with 2-ethyl-1-hexylamine afforded the monomer EHNI, which was used for the synthesis of the reference polymer polEHNI and as comonomer for the rod-containing statistical copolymers.

The synthesis of the first aramid-carrying monomer with a solubilizing alkyl chain attached to the aramid segment started with the protection of the carboxylic acid of 4-aminosalicylic acid as a methyl ester, which afforded compound 1 (Scheme 1). A 2-ethylhexyl side chain was introduced via Williamson ether synthesis between the aromatic hydroxyl group and a haloalkane to give 2. After saponification of methyl ester 2 into the free carboxylic acid 3, a condensation with cis-5-norbornene-exo-2,3-dicarboxylic anhydride yielded the imide 4 . This was followed by consecutive amide bond formations using thionyl chloride in $\mathrm{N}$-methyl-2-pyrrolidone (NMP) solution to afford monoEtHexROD (Scheme 1). Due to intramolecular hydrogen bond formation between the amide $\mathrm{N}-\mathrm{H}$ donor and the alkyloxy O-acceptor, no intermolecular aggregation of this trimer via hydrogen bonds is possible. However, the intramolecular hydrogen bond formation allows neighbouring phenyl rings to be perfectly coplanar, thereby "flattening" the trimer and allowing greater dispersion force induced stabilization due to the flat rigid shape. ${ }^{17}$

The second aramid-carrying monomer, designed to be able to undergo H-bonding, needed a solubilizing feature to facilitate its synthesis. Thus, in order to prevent H-bonds during

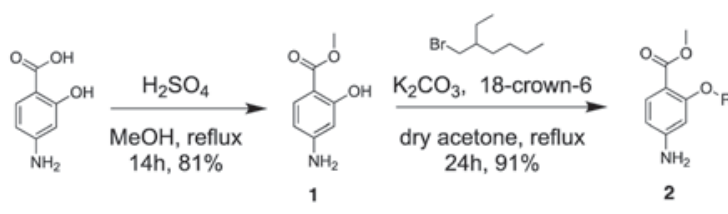

2
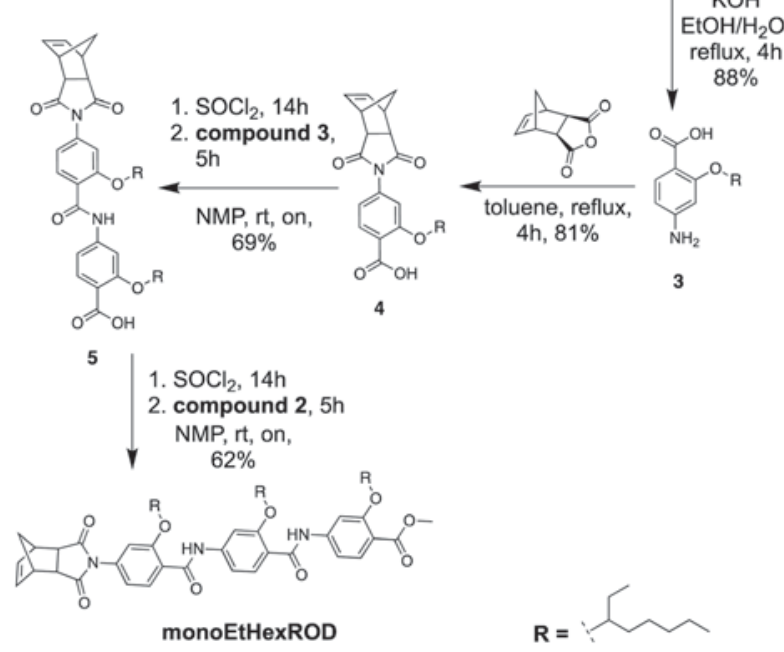

Scheme 1 Multistep synthesis of the ethylhexyl-rod monomer (monEtHexROD). synthesis and enable good solubility in non-polar solvents, an $\mathrm{N}$-dimethoxybenzyl (DMB) protecting group was attached to the amide linkage, which changes the configuration of the amide bond from trans into cis (with respect to the phenyl rings). The synthesis started with the methylation of 4-aminobenzoic acid to afford methyl ester 6 (Scheme 2), which was reacted with 2,4-dimethoxybenzaldehyde to afford the secondary amine 7 through reductive amination. Subsequent saponification gave the free carboxylic acid 8. Finally, the aramid units were consecutively attached in the same manner as for monoEtHexROD (Scheme 1) to yield monomer monoDMBrod (Scheme 2).

The polymerizations were carried out by fast injection of a degassed dichloromethane (DCM) solution of initiator G1 (Grubbs' 1st generation catalyst, dichloro(benzylidene)-bis (tricyclohexylphosphine)ruthenium(II)) to a degassed DCM solution of either a mixture of monomers EHNI and monoEtHexROD (Scheme 3) or a mixture of ENHI and monoDMBrod (Scheme 4). In both series, the composition was systematically varied and the fraction of the rod-containing monomers was chosen to be 10, 25, 35 and 50 molar percent. The rod percentages are reported in the polymer acronym as P10, P20, P35, and P50 respectively, while the polymers with different type of rod are named according to their rod monomer such as polEtHexROD and polDMBrod, which
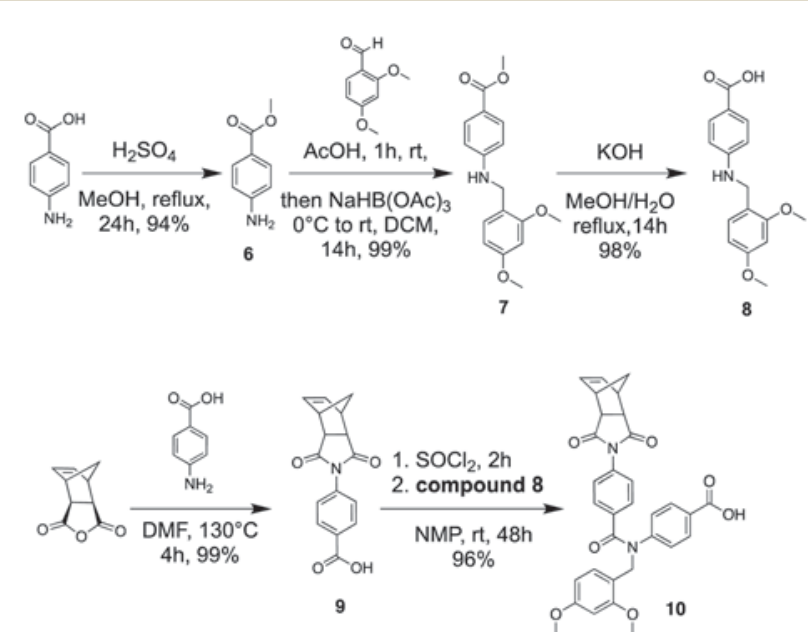

$\mathrm{SOCl}_{2}, 2 \mathrm{~h}$

2. compound 7 NMP, rt, 48h

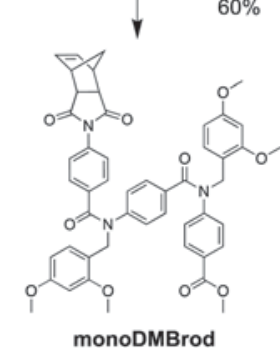

Scheme 2 Multistep (monoDMBrod). 


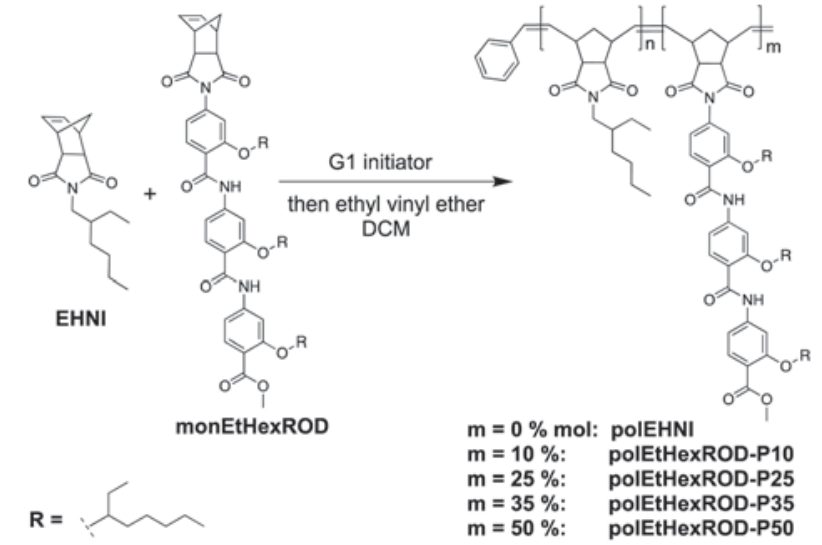

Scheme 3 Synthesis of polEHNI and copolymers of EHNI and different mol percentages of monoEtHexROD.

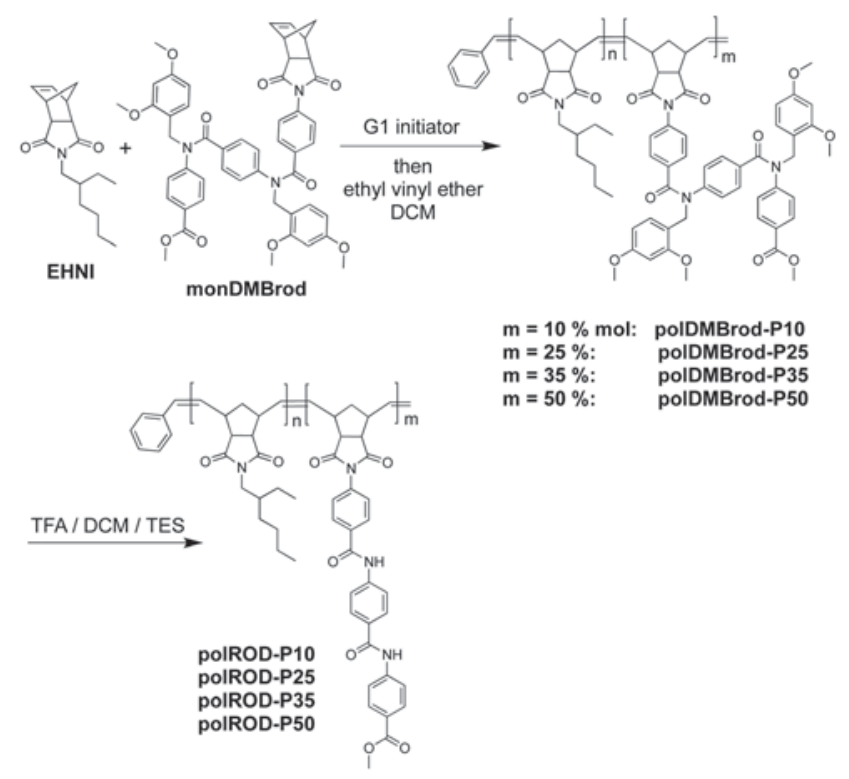

Scheme 4 Synthesis of copolymers containing EHNI and different mol percentages of DMB-protected rod monomer and DMB-deprotection of the copolymers.

correspond to monoEtHexROD and monoDMBrod respectively. Finally, polROD corresponds to the deprotected form of polDMBrod. A reference polymer with only EHNI (polEHNI) was also prepared.

Gel Permeation Chromatography/Size Exclusion Chromatography (GPC/SEC) traces of all polymers prepared are shown in Fig. 1. The GPC traces show narrow peaks with molecular weight dispersities between $D=1.1$ and 1.2 for the reference polymer polEHNI and both copolymer series, polDMBrod and polEtHexROD. However, as can be seen in Fig. 1a and c, the molecular weight decreases with increasing rod content. ${ }^{1} \mathrm{H}$-NMR data indicates that the desired molar ratio between either monDMBrod or monEtHexROD and EHNI monomer is identical to the monomer feed ratio. This demonstrates that
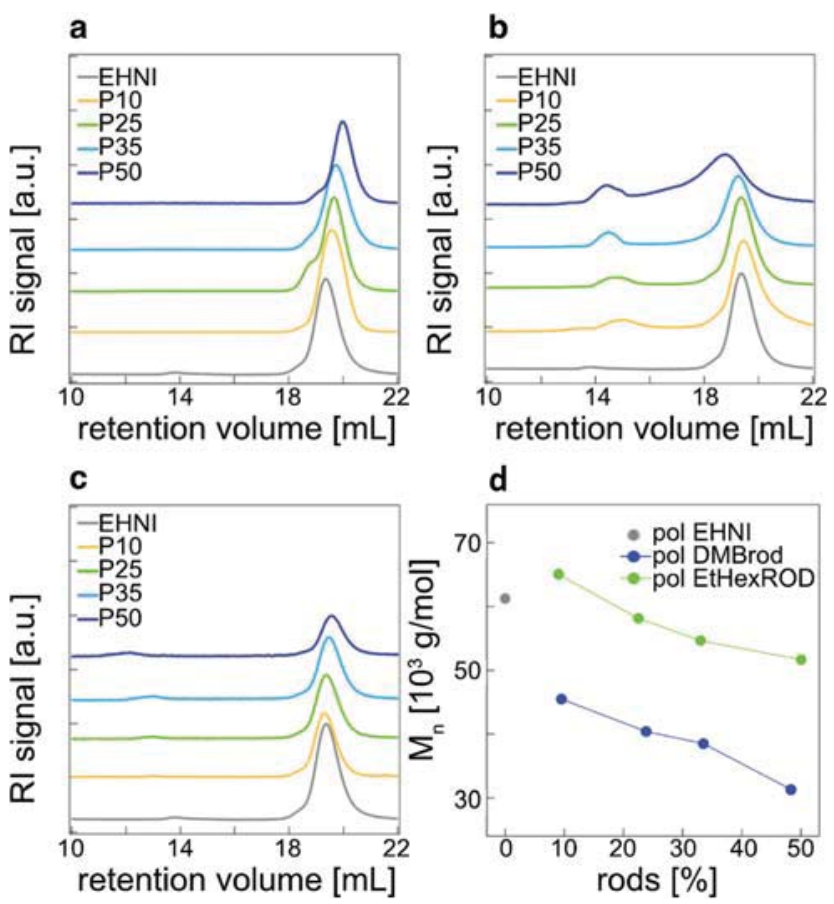

Fig. 1 GPC elution curves (solvent: THF, calibration: polystyrene standards, detector: refractive index) of polymer samples: (a) DMB-protected rods (polDMBrod) (b) DMB-deprotected rigid side chains (polROD), (c) ethylhexyl ether rod polymers (polEtHexROD) and (d) influence of the rod content on the number-average molecular weight. Data of polEHNI are also shown.

the steric hindrance of the rods might slow down the reaction, but it does not interfere with the relative reactivity of the two monomers. Furthermore, GPC traces for the DMB-deprotected polROD polymers show an increase of the molecular weight due to aggregation via H-bonds in THF (see Fig. 1b). These measurements are supported by dynamic light scattering (DLS) experiments in THF (Table 1, and Fig. S2†). Both polDMBrod and polEtHexROD polymers show hydrodynamic radii $r_{\mathrm{H}}$ between 6 and $10 \mathrm{~nm}$, indicating good solvation and no aggregate formation. On the other hand, polymers polROD-P10 and polROD-P25 show hydrodynamic radii of 211 and $522 \mathrm{~nm}$, respectively, even at low rod concentration, indicating strong aggregation. For polROD-P35 and polROD-P50 DLS was impossible, due to the insolubility of these polymers in THF caused by strong aggregation.

All polymers were further analysed by thermogravimetric analysis (TGA) and differential scanning calorimetry (DSC). Fig. $2 \mathrm{a}-\mathrm{c}$ show the results of the thermogravimetric analysis of all polymers under nitrogen atmosphere. In general, the TGA traces do not indicate a very significant change in thermal stability, regardless of the copolymer composition, and the decomposition onset temperatures are comparable. No degradation can be detected until $400{ }^{\circ} \mathrm{C}$, even for the polEHNI homopolymer. At higher temperatures, a continuous decomposition occurred for all compounds, as expected for random/ statistical copolymers. ${ }^{18}$ A hypothetical block copolymer for- 
Table 1 Number-average molecular weight $\left(M_{n}\right)$ and dispersity $(D)$ derived from GPC data (solvent: THF, calibration: polystyrene standards), DLS data (0.5 $\mathrm{mg} \mathrm{mL}^{-1}$ THF solutions), experimental rod content calculated from NMR integrals, and the glass transition temperature $\left(T_{\mathrm{g}}\right)$ established by DSC measurements

\begin{tabular}{lccllr}
\hline & $\begin{array}{c}M_{\mathrm{n}} \times 10^{3} \\
{\left[\mathrm{~g} \mathrm{~mol}^{-1}\right]}\end{array}$ & $\Xi$ & $r_{\mathrm{H}}[\mathrm{nm}]$ & $\operatorname{Rod}[\%]$ & $T_{\mathrm{g}}\left[{ }^{\circ} \mathrm{C}\right]$ \\
\hline Polymer & 61 & 1.1 & 10 & 0 & 72 \\
polEHNI & 46 & 1.1 & 10 & 9.5 & 91 \\
polDMBrod-P10 & 40 & 1.1 & 9 & 23.8 & 115 \\
polDMBrod-P25 & 39 & 1.2 & 8 & 33.5 & 123 \\
polDMBrod-P50 & 31 & 1.1 & 6 & 48.3 & 129 \\
polROD-P10 & 54 & 1.2 & 211 & - & 104 \\
polROD-P25 & 61 & 2.6 & 522 & - & 147 \\
polROD-P35 & 100 & 2.9 & $\mathrm{~N} / \mathrm{A}$ & - & 171 \\
polROD-P50 & 218 & 2.4 & $\mathrm{~N} / \mathrm{A}$ & - & 201 \\
polEtHexROD-P10 & 65 & 1.2 & 7 & 9.0 & 82 \\
polEtHexROD-P25 & 58 & 1.2 & 7 & 22.5 & 98 \\
polEtHexROD-P35 & 55 & 1.2 & 7 & 33.9 & 106 \\
polEtHexROD-P50 & 52 & 1.2 & 7 & 50.0 & 118
\end{tabular}
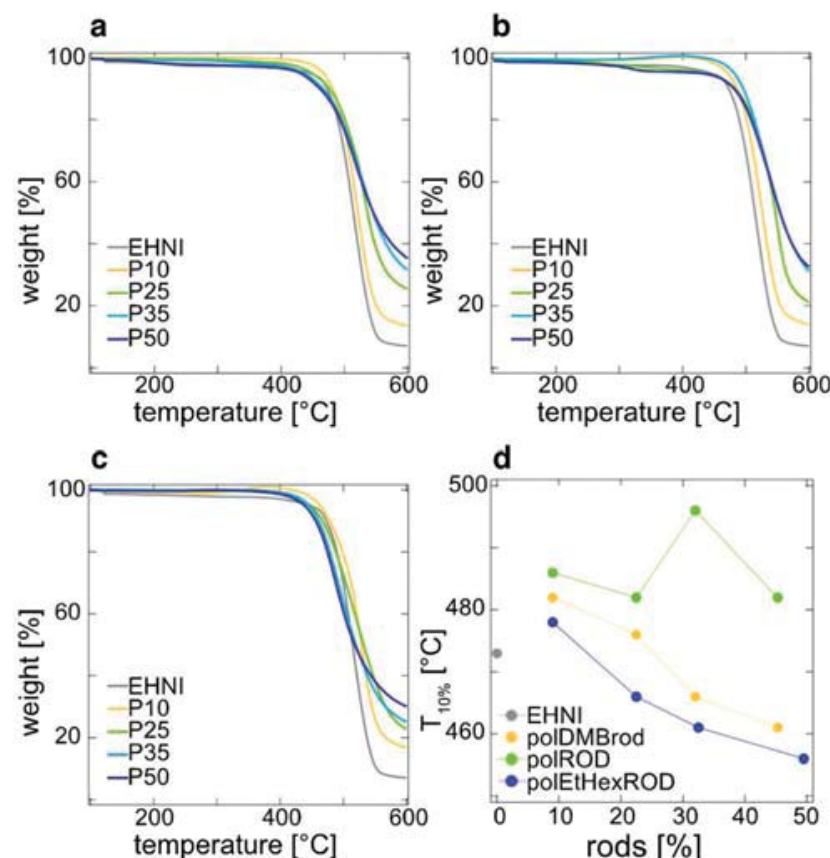

$$
\text { d }
$$

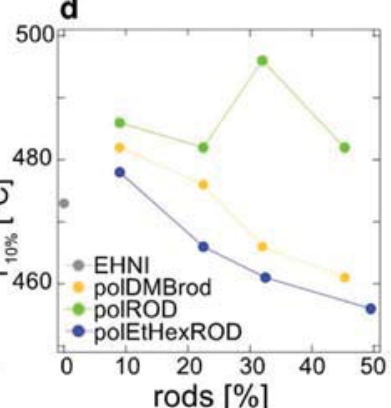

Fig. 2 Thermogravimetric analysis traces of polymer samples: (a) DMBprotected (polDMBrod), (b) DMB-deprotected (polROD), (c) ethylhexyl ether carrying rod polymers (polEtHexROD) and (d) temperatures of $10 \%$ weight loss as function of rod content.

mation would be indicated by a step-wise weight loss, which is, however, not observed. Comparing the temperatures at $10 \%$ weight loss, $T_{10 \%}$, a difference in the decomposition behaviour of the different rods incorporated in the polymer can be observed. The DMB-deprotected polROD show the highest $T_{10 \%}$ values, followed by the DMB-protected polDMBrod, and the lowest temperature $T_{10 \%}$ are seen for polEtHexROD (Fig. 2d). It has to be noted that the thermostability decreases with the augmentation of the rod concentration for
PolDMBrod and polEtHexROD. This is attributed mainly to the loss of DMB-protecting group and the ethyl hexyl side chain for both polymers respectively.

These results imply that the interactions between rods have a positive influence on thermostability of the material, when hydrogen bonds are present. However, these differences are relatively small due to the fact, that the backbone of polEHNI already shows a high thermal stability. TGA data also show an increasing residual mass fraction at $600{ }^{\circ} \mathrm{C}$ with higher rod content, indicating that the remaining mass consisted of the more stable aromatic side chains.

To obtain a better understanding of the influence of different rods on the thermal properties of the polymers, DSC measurements were performed. The results are illustrated in the graphs shown in Fig. 3a-c. Since the TGA measurements reveal that all polymers are stable below $300{ }^{\circ} \mathrm{C}$, DSC measurements were performed between $-80{ }^{\circ} \mathrm{C}$ and $300{ }^{\circ} \mathrm{C}$. Homopolymer polEHNI exhibits a glass transition temperature $\left(T_{\mathrm{g}}\right)$ of $72{ }^{\circ} \mathrm{C}$. The $T_{\mathrm{g}}$ of the copolymers predominantly depends on the concentration, but also on the nature of the attached rod, and increases to up to $201{ }^{\circ} \mathrm{C}$ for the compositions investigated. Plots of $T_{\mathrm{g}} v s$. the rod content (see Fig. 3d) clearly show a linear dependence for both, polROD and polEtHexROD. The slope of the function, and hence the influence of rod content, is higher for polROD. This indicates that H-bonding between the amide groups shows a greater influence on $T_{\mathrm{g}}$, than dispersion forces between flat rod-like
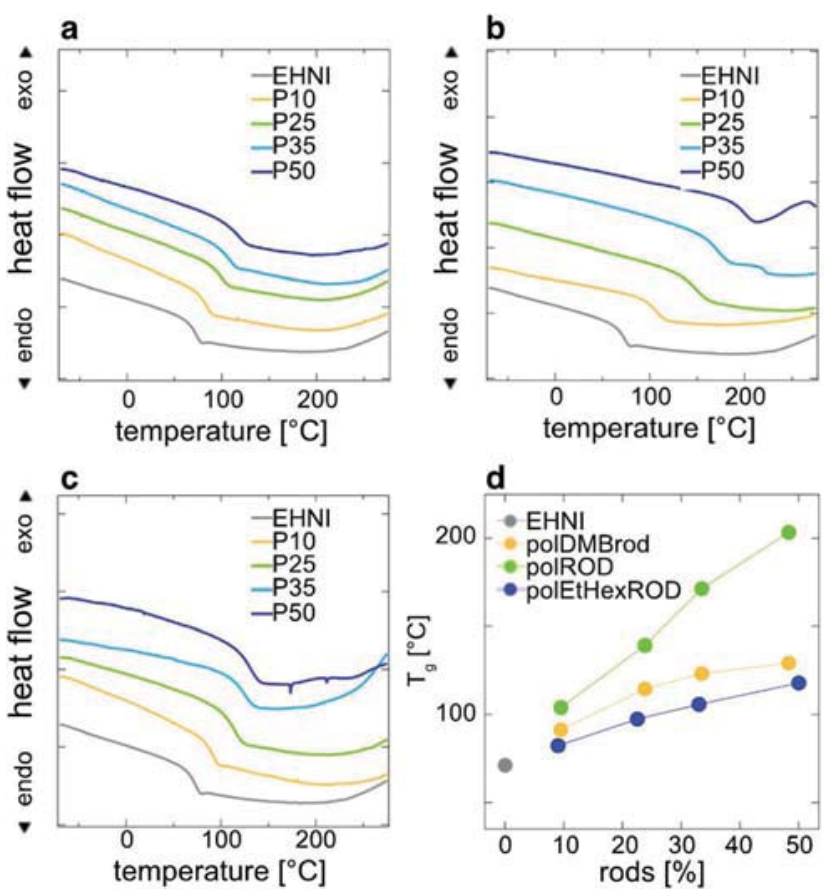

Fig. 3 Traces of the second heating in DSC analysis of polymer samples: (a) DMB-protected (polDMBrod), (b) DMB-deprotected (polROD) and (c) ethylhexyl ether rod carrying polymers (polEtHexROD). Graph (d) is indicating the dependence of $T_{\mathrm{g}}$ on the rod content. For full heating-cooling cycles see Fig. S6. $\dagger$ 
molecules in the case of polEtHexROD. This behaviour is possibly due to the reduction of internal plasticization. ${ }^{19}$ By decreasing the amount of the flexible EHNI spacers, the rigid components are closer to each other and hence interactions are more likely to occur. Furthermore, it must be noted, that the increasing molecular weight does not significantly affect the $T_{\mathrm{g} .}{ }^{20,21}$

Annealing the polymers at $15{ }^{\circ} \mathrm{C}$ above their respective $T_{\mathrm{g}}$ did not affect the $T_{\mathrm{g}}$ for polROD samples (Fig. S7 $\dagger$ ). Furthermore, annealing did not lead to crystallization, confirming the expectation that these copolymers are fully amorphous. $^{22}$ One can speculate that an increase of the rod content would lead to a further increase of $T_{\mathrm{g}}$. However, higher percentages of the rigid side chains would most likely also lead to insolubility and such compositions were thus not investigated.

After investigation of the thermal characteristics, attention was paid to the mechanical properties of the polymers. For this purpose, polymer films were produced by compression molding in a hot-press. Films with a thickness of $0.12 \mathrm{~mm} \pm$ $0.01 \mathrm{~mm}$ were produced. Unfortunately, films made from polymers with a rod-content of more than $10 \%$ proved to be too brittle to handle. Thus, only the mechanical properties of polEHNI, polDMBrod-P10, polROD-P10, and polEtHexROD-P10 could be compared. ESI Fig. S8 and $\mathrm{S} 9 \dagger$ show the stress-strain curves and Table 2 lists the maximum stress $\left(\sigma_{\max }\right)$, elongation at maximal stress $(\varepsilon)$, the Young's moduli $(E)$ and the storage modulus $\left(E^{\prime}\right)$ of the respective polymers.

All polymers tested display ultimately very similar mechanical properties. An elongation at maximal force of the order of $3 \%$ is observed for all polymers. Some of the stress-strain curves appear to show some yielding (ESI Fig. S9†), and the maximum strain at break was in all cases lower than $20 \%$. Unfortunately, perhaps due to sample inhomogenities, the tensile test results show considerable variations (ESI Fig. S9†), and no clear trend with respect to the elongation at break could be established. On the other hand, the introduction of the aramids appears to lead to a slight increase of the tensile strength $\sigma_{\max }$. Indeed, amongst all samples investigated, polROD-P10 showed the highest $\sigma_{\max }$, which is attributed to $\mathrm{H}$-bonding effecting the tensile strength beneficially compared to polEHNI. For polEtHexROD-P10 a decrease of $\sigma_{\max }$ was observed, indicating that monEtHexROD were not able to interact via $\mathrm{H}$-bonds but rather disturb the internal structure of the backbone. polDMBrod and polROD also display the highest Young's moduli (1.5 and 1.6 GPa, respectively), but again, the differences between the compositions are very small.

To get an insight into the thermal dependence of the mechanical properties, temperature dependent dynamic mechanical analysis (DMA) experiments were conducted. The DMA traces (Fig. 4) reveal a rigid, glassy regime, with a roomtemperature storage modulus $E^{\prime}$ that increases from $1.3 \mathrm{GPa}$ (polEHNI) to 1.6 GPa for polROD-P10 This is clearly indicating the beneficial effect of $\mathrm{H}$-bonds on the $E^{\prime}$ modulus. For polDMBrod and polEtHexROD no improved storage moduli were obtained, as indicated by similar DMA traces as for polEHNI. The DMA traces also clearly confirm an increase of the $T_{\mathrm{g}}$, which increases from $72{ }^{\circ} \mathrm{C}$ (polEHNI) to $82{ }^{\circ} \mathrm{C}$ (polEtHexROD-P10) to $91{ }^{\circ} \mathrm{C}$ (polDMBrod-P10) to $104{ }^{\circ} \mathrm{C}$ polROD-P10. Thus, the DMA data confirm the DSC results and indicate that the introduction of $\mathrm{H}$-bonding monomers has the largest influence on $T_{\mathrm{g}}$.

Powder X-ray diffraction (XRD) experiments were performed, in order to get insight in the molecular organization of the side chains within the polymer. Diffraction patterns are shown in Fig. 5. For polEHNI two diffuse scattering halos at $2 \theta=5^{\circ}$ and $2 \theta=17.5^{\circ}$ are present. Even though these broad, diffuse peaks show some degree of organization, the absence of sharp Bragg peaks reveals that polEHNI shows no crystallinity. Upon increasing the rod content in both series, polEtHexROD and polROD, an incremental decrease of the intensity of the diffuse peaks was observed. This effect was attributed to the interference of the bulky rod side chains with

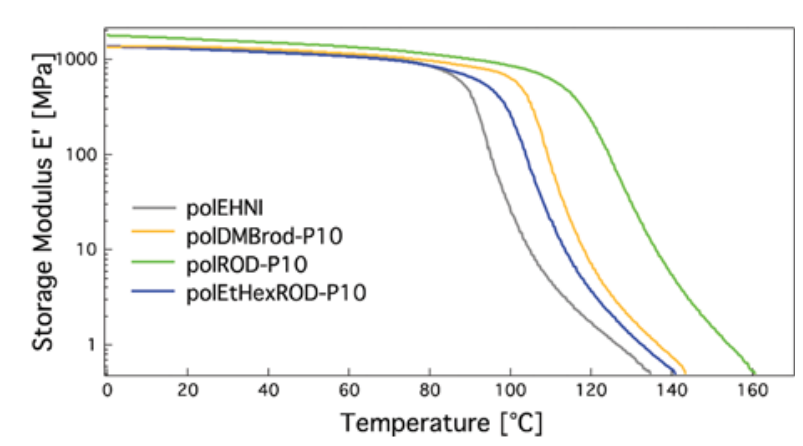

Fig. 4 Temperature dependence of storage modulus $E^{\prime}$ obtained via DMA experiments on $0.12 \pm 0.01 \mathrm{~mm}$ hot-pressed films of polymer samples.

Table 2 Mechanical properties determined via different DMA experiment of all compositions suitable for mechanical analysis (higher rod-content polymers were to brittle)

\begin{tabular}{|c|c|c|c|c|}
\hline Polymer & $\begin{array}{l}\text { Tensile strength } \sigma_{\max } \\
{[\mathrm{MPa}]}\end{array}$ & $\begin{array}{l}\text { Elongation at maximal stress } \varepsilon_{\max } \\
{[\%]}\end{array}$ & $\begin{array}{l}\text { Young's modulus } E \\
{[\mathrm{GPa}]}\end{array}$ & $\begin{array}{l}\text { Storage modulus } E^{\prime}\left(25^{\circ} \mathrm{C}\right) \\
{[\mathrm{GPa}]}\end{array}$ \\
\hline polEHNI & 31 & 3.3 & 1.3 & 1.3 \\
\hline polDMBrod-P10 & 34 & 3.1 & 1.6 & 1.2 \\
\hline polROD-P10 & 37 & 3.0 & 1.5 & 1.6 \\
\hline polEtHexROD-P10 & 26 & 2.7 & 1.2 & 1.2 \\
\hline
\end{tabular}



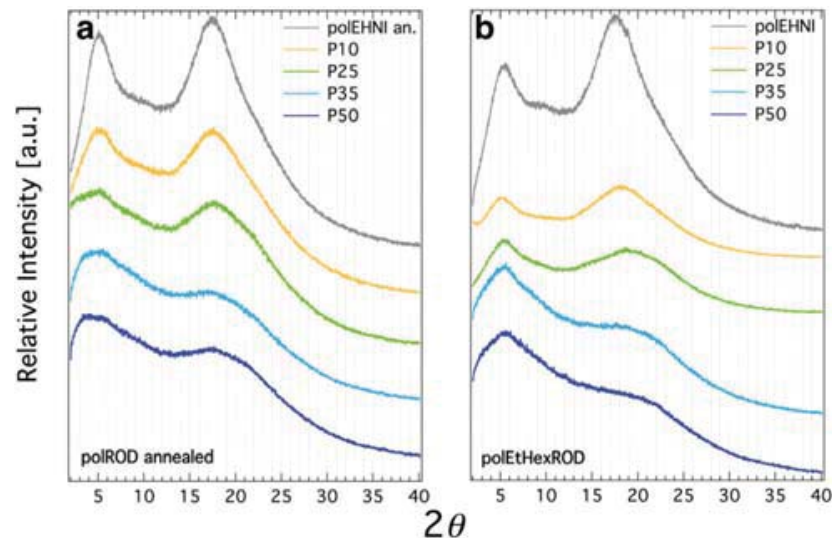

Fig. 5 Powder X-ray diffraction profiles at $25^{\circ} \mathrm{C}$ for different rod containing samples: (a) polROD annealed $15^{\circ} \mathrm{C}$ above their respective $T_{\mathrm{g}}$ and (b) ethylhexyl rod carrying polymers polEtHexROD. Graphs presented with intensity offset for clarity.

the organization of ethylhexyl side groups in polEHNI and the related spacing of the backbone. It must be noted, that annealing at $15{ }^{\circ} \mathrm{C}$ above $T_{\mathrm{g}}$ of polROD samples was necessary in order to observe this trend. Based on X-ray diffraction data, it can be concluded, that all different polymers show amorphous behaviour.

Interestingly, even though polEHNI did not show real crystallinity, some birefringence was observed by cross-polarized light microscopy due to organization of the backbone to some extent (POM, ESI Fig. S12 $\dagger$ ). Also, the POM images of precipitated copolymers show some birefringence, which, however, decreased with increasing rod content (Fig. S11-S13†). In order to investigate if the rod-like side chains may serve as mesogens and if polymers with higher rod content would show liquid crystalline behaviour at elevated temperatures, POM was also performed under heating the samples (to $15^{\circ} \mathrm{C}$ above their respective $T_{\mathrm{g}}$ ). However, heating the polymers extinguished the birefringence entirely, as shown in ESI Fig. S11-S13. $\dagger$ The same result was observed, when high-rodcontent polymers polROD-P35 and polROD-P50 were annealed $15{ }^{\circ} \mathrm{C}$ above their respective $T_{\mathrm{g}}$ and cooled down very slowly (ESI Fig. S13. $\dagger$ Additionally, DSC curves of these samples showed no exothermic peaks, indicating no crystalline behaviour (ESI Fig. S7†). In summary, all observations made by means of POM support X-ray measurements. Initial organization (not crystallinity) of polEHNI was inhibited by introduction of rigid rod side groups into the polymers.

\section{Conclusion}

Two series of new comb-like poly(norbornene)s carrying lateral rod-like aramid group were synthesized in which the aramid moieties display different types of non-covalent interactions. The results show that the glass transition temperature increases proportionally with the concentration of the $\mathrm{H}$-bonding monomer. All polymers showed a $T_{\mathrm{g}}$ above room temperature, which renders the materials glassy and brittle. The high $T_{\mathrm{g}}$ also provides a high stiffness and high temperature stability. It was found, that the brittleness of the material increases with increasing rod content; in fact, materials with a rod content of higher than $10 \%$ were too brittle for mechanical analysis. DSC analyses indicated that all polymers are amorphous, which is supported by X-ray diffraction before and after annealing. Investigations of their material properties showed an increasing $T_{\mathrm{g}}$ due to the $\mathrm{H}$-bond interaction between the aramid rods and a linear increase of $T_{\mathrm{g}}$ proportionally to the rod percentage. Hence, DMB-deprotected rod polymers showed overall the highest $T_{\mathrm{g}}$ according to their rod percentages. This means the hydrogen bond interaction within the polymer are stronger than the $\pi-\pi$-stacking carried by EtHexROD polymers as expected. High $T_{\mathrm{g}}$ polymers are of interest due to their thermal stability. However, most of these materials are difficult to handle due to the heat required to be processed. These rod-containing polymers are interesting because they could be processed in solution at lower temperature than their respective $T_{\mathrm{g}}$. These results showed that aramid rods could be used for the synthesis of polymers with tailormade thermal behaviour as it is the case for heat resistant and/or fire-retardant materials.

\section{Conflicts of interest}

There are no conflicts to declare.

\section{Acknowledgements}

PK, SD, SS, CW and AFMK thank the National Center for Competence in Research (NCCR) "Bio-inspired Materials" and the Swiss National Science Foundation for financial support. Furthermore, the authors thank the following people for support: Worarin Meesorn (DMA experiments, Adolphe Merkle Institute), Aurelién Crochet (powder X-ray, University of Fribourg) and Sandra Graterol (DSC, Adolphe Merkle Institute).

\section{Notes and references}

1 P. W. Morgan, Macromolecules, 1977, 10, 1381-1390.

2 M. G. Northolt and J. J. van Aartsen, J. Polym. Sci., Polym. Lett. Ed., 1973, 11, 333-337.

3 M. G. Northolt, Eur. Polym. J., 1974, 10, 799-804.

4 D. Tanner, J. A. Fitzgerald and B. R. Phillips, Angew. Chem., Int. Ed. Engl., 1989, 28, 649-654.

5 H. Seyler, E. Berger-Nicoletti and A. F. M. Kilbinger, J. Mater. Chem., 2007, 17, 1954-1957.

6 M. Schulze, B. Michen, A. Fink and A. F. M. Kilbinger, Macromolecules, 2013, 46, 5520-5530.

7 H. Seyler and A. F. M. Kilbinger, Macromolecules, 2009, 42, 9141-9146. 
8 H. Seyler and A. F. M. Kilbinger, Macromolecules, 2010, 43, 5659-5664.

9 V. Shibaev, A. Bobrovsky and N. Boiko, Prog. Polym. Sci., 2003, 28, 729-836.

10 K. O. Kim and T. L. Choi, ACS Macro Lett., 2012, 1, 445-448.

11 M. Saariaho, A. Subbotin, I. Szleifer, O. Ikkala and G. Ten Brinke, Macromolecules, 1999, 32, 4439-4443.

12 T. Kato, J. Uchida, T. Ichikawa and B. Soberats, Polym. J., 2018, 50, 149-166.

13 Y. F. Zhu, Z. Y. Zhang, Q. K. Zhang, P. P. Hou, D. Z. Hao, Y. Y. Qiao, Z. Shen, X. H. Fan and Q. F. Zhou, Macromolecules, 2014, 47, 2803-2810.

14 Y. Zhang, R. Cao, J. Shen, C. S. F. Detchou, Y. Zhong, H. Wang, S. Zou, Q. Huang, C. Lian, Q. Wang, J. Zhu and B. Gong, Org. Lett., 2018, 20, 1555-1558.
15 C. A. Hunter and J. K. M. Sanders, J. Am. Chem. Soc., 1990, 112, 5525-5534.

16 C. A. Hunter, K. R. Lawson, J. Perkins and C. J. Urch, J. Chem. Soc., Perkin Trans. 2, 2001, 5, 651-669.

17 C. R. Martinez and B. L. Iverson, Chem. Sci., 2012, 3, 21912201.

18 C. De Ruijter, W. F. Jager, J. Groenewold and S. J. Picken, Macromolecules, 2006, 39, 3824-3829.

19 T. Hu, J. Yi, J. Xiao and H. Zhang, Polym. J., 2010, 42, 752758.

20 T. G. Fox and P. J. Flory, J. Polym. Sci., 1954, 14, 315-319.

21 Z. Komiya, C. Pugh and R. R. Schrock, Macromolecules, 1992, 25, 3609-3616.

22 G. Mao, J. Wang, S. R. Clingman, C. K. Ober, J. T. Chen and E. L. Thomas, Macromolecules, 1997, 30, 2556-2567. 